\title{
Stretched exponential relaxation in the mode-coupling theory for the Kardar-Parisi-Zhang equation
}

\author{
Francesca Colaiori and M. A. Moore \\ Department of Physics and Astronomy, University of Manchester, Manchester, M13 9PL, United Kingdom
}

(November 20, 2018)

\begin{abstract}
We study the mode-coupling theory for the Kardar-Parisi-Zhang equation in the strong-coupling regime, focusing on the long time properties. By a saddle point analysis of the mode-coupling equations, we derive exact results for the correlation function in the long time limit - a limit which is hard to study using simulations. The correlation function at wavevector $\mathbf{k}$ in dimension $d$ is found to behave asymptotically at time $t$ as $C(\mathbf{k}, t) \simeq \frac{A}{k^{d+4-2 z}}\left(B t k^{z}\right)^{\gamma / z} e^{-\left(B t k^{z}\right)^{1 / z}}$, with $\gamma=(d-1) / 2$, $A$ a determined constant and $B$ a scale factor.

PACS numbers: 05.40.-a, 64.60.Ht, 05.70Ln, 68.35.Fx
\end{abstract}

The Kardar-Parisi-Zhang (KPZ) [1] equation is a simple non-linear Langevin equation, proposed in 1986 as a coarse grained description of a growing interface. Probably due to the fact that is the simplest generalization of the diffusion equation which includes a relevant nonlinear term, the KPZ equation also arises in connection with many other important physical problems (the Burgers equation for one-dimensional turbulence [2], directed polymers in a random medium [3 5] etc.).

The KPZ equation for a growing interface, described by a single valued height function $h(\mathbf{x}, t)$ on a $d$ dimensional substrate $\mathbf{x} \in \Re^{d}$ is:

$$
\partial_{t} h(\mathbf{x}, t)=\nu \nabla^{2} h+\frac{\lambda}{2}(\nabla h)^{2}+\eta(\mathbf{x}, t) .
$$

The first term represents the surface tension forces which tend to smooth the interface, the second describes the nonlinear growth locally normal to the surface, and the last is a noise which mimics the stochastic nature of the growth process [6]. We choose the noise to be Gaussian, with zero mean and second moment $\left\langle\eta(\mathbf{x}, t) \eta\left(\mathbf{x}^{\prime}, t^{\prime}\right)\right\rangle=$ $2 D \delta^{d}\left(\mathbf{x}-\mathbf{x}^{\prime}\right) \delta\left(t-t^{\prime}\right)$. The steady state interface profile is usually described in terms of the roughness: $w=$ $\left\langle h^{2}(\mathbf{x}, t)\right\rangle-\langle h(\mathbf{x}, t)\rangle^{2}$ which for a system of size $L$ behaves like $L^{\chi} f\left(t / L^{z}\right)$, where $f(x) \rightarrow$ const as $x \rightarrow \infty$ and $f(x) \sim x^{\chi / z}$ as $x \rightarrow 0$, so that $w$ grows with time like $t^{\chi / z}$ until it saturate to $L^{\chi}$ when $t \sim L^{z}$. $\chi$ and $z$ are the roughness and dynamic exponent respectively.

The phenomenology of the KPZ is well known: above two dimensions, there are two distinct regimes, separated by a critical value $\lambda_{c}$ of the non-linearity coefficient. In the weak coupling regime $\left(\lambda<\lambda_{c}\right)$ the non-linear term is irrelevant and the behavior is governed by the Gaus$\operatorname{sian}(\lambda=0)$ fixed point. The KPZ in this regime is equivalent to the linear Edward Wilkinson equation, for which the exponents are known exactly $\chi=(2-d) / 2$ and $z=2$. The more challenging strong coupling regime $\left(\lambda>\lambda_{c}\right)$, where the non-linearity is relevant is characterised by anomalous exponents, which are not known exactly in general dimension $d$. From the Galilean in- variance [2] (invariance of Eq. (11) under an infinitesimal tilting of the surface) one can derive the relation $\chi+z=2$, which leaves just one independent exponent. For the special case when $d=1$, the existence of a fluctuation-dissipation theorem gives the exact results $\chi=1 / 2, z=3 / 2$.

We take a self-consistent approach, the mode-coupling (MC) approximation [2], in which in the diagrammatic expansion for the correlation and response function only diagrams which do not renormalise the three point vertex $\lambda$ are retained. The MC approximation has been remarkably successful in other areas of condensed matter physics, for example in the study of structural glass transitions [7], binary mixtures [8] and critical dynamics of magnets [8,9]. KPZ mode-coupling reproduces the exact values of the exponents in $d=1$. Furthermore MC equations have been shown to arise from the large $N$-limit of an generalised $N$-component KPZ equation [10], which allows in principle a systematic approach to the theory beyond mode coupling, by expanding in $1 / N$.

Our study focuses on the large time properties of the KPZ equation: we predict a stretched exponential decay for the correlation function at large times. A phenomenological stretched exponential law was used as long ago as 1854 to fit electronic relaxation data for a Leiden jar capacitor 11], and then been rediscovered many times: fitting functions involving stretched exponentials are nowadays widely used in phenomenogical analysis of relaxation data (examples are dielectric relaxation [12] and glassy relaxation [7, 13]). However, only a few analytical arguments [14] are able to reproduce stretched exponential relaxation in complex systems. Our prediction is in principle amenable of numerical verification, even though usual numerical techniques, mainly based on simulations 15] of discrete microscopical models which belong to the KPZ universality class [16], are hard in the long time asymptotic region.

Mode-coupling equations are coupled equation for the correlations and response function. The correlation and response function are defined in Fourier space by 


$$
\begin{gathered}
C(\mathbf{k}, \omega)=\left\langle h(\mathbf{k}, \omega) h^{*}(\mathbf{k}, \omega)\right\rangle \\
G(\mathbf{k}, \omega)=\delta^{-d}\left(\mathbf{k}-\mathbf{k}^{\prime}\right) \delta^{-1}\left(\omega-\omega^{\prime}\right)\left\langle\frac{\partial h(\mathbf{k}, \omega)}{\partial \eta\left(\mathbf{k}^{\prime}, \omega^{\prime}\right)}\right\rangle
\end{gathered}
$$

where $\langle\cdot\rangle$ indicate an average over $\eta$. In the mode-coupling approximation, the correlation and response functions are the solutions of two coupled equations,

$$
\begin{gathered}
G^{-1}(\mathbf{k}, \omega)=G_{0}^{-1}(\mathbf{k}, \omega)+\lambda^{2} \int \frac{d \Omega}{2 \pi} \int \frac{d^{d} q}{(2 \pi)^{d}}[\mathbf{q} \cdot(\mathbf{k}-\mathbf{q})][\mathbf{q} \cdot \mathbf{k}] G(\mathbf{k}-\mathbf{q}, \omega-\Omega) C(\mathbf{q}, \Omega), \\
C(\mathbf{k}, \omega)=C_{0}(\mathbf{k}, \omega)+\frac{\lambda^{2}}{2}|G(\mathbf{k}, \omega)|^{2} \int \frac{d \Omega}{2 \pi} \int \frac{d^{d} q}{(2 \pi)^{d}}[\mathbf{q} \cdot(\mathbf{k}-\mathbf{q})]^{2} C(\mathbf{k}-\mathbf{q}, \omega-\Omega) C(\mathbf{q}, \Omega),
\end{gathered}
$$

where $G_{0}(\mathbf{k}, \omega)=\left(\nu k^{2}-i \omega\right)^{-1}$ is the bare response function, and $C_{0}(\mathbf{k}, \omega)=2 D|G(\mathbf{k}, \omega)|^{2}$. In the strong coupling limit, $G(\mathbf{k}, \omega)$ and $C(\mathbf{k}, \omega)$ take the following scaling forms

$$
\begin{gathered}
G(\mathbf{k}, \omega)=k^{-z} g\left(\omega / k^{z}\right), \\
C(\mathbf{k}, \omega)=k^{-(2 \chi+d+z)} n\left(\omega / k^{z}\right),
\end{gathered}
$$

and Eqs. (2) and (3) translate into the following coupled equations for the scaling functions $n(x)$ and $g(x)$ :

$$
\begin{gathered}
g^{-1}(x)=-i x+I_{1}(x), \\
n(x)=|g(x)|^{2} I_{2}(x),
\end{gathered}
$$

where $x=\omega / k^{z}$ and $I_{1}(x)$ and $I_{2}(x)$ are given by

$$
\begin{gathered}
I_{1}(x)=P \int_{0}^{\pi} d \theta \sin ^{d-2} \theta \int_{0}^{\infty} d q \cos \theta(\cos \theta-q) q^{2 z-3} r^{-z} \int_{-\infty}^{\infty} d y g\left(\frac{x-q^{z} y}{r^{z}}\right) n(y), \\
I_{2}(x)=\frac{P}{2} \int_{0}^{\pi} d \theta \sin ^{d-2} \theta \int_{0}^{\infty} d q(\cos \theta-q)^{2} q^{2 z-3} r^{-(d+4-z)} \int_{-\infty}^{\infty} d y n\left(\frac{x-q^{z} y}{r^{z}}\right) n(y),
\end{gathered}
$$

with $P=\lambda^{2} /\left(2^{d} \Gamma\left(\frac{d-1}{2}\right) \pi^{(d+3) / 2}\right), r^{2}=1+q^{2}-2 q \cos \theta$. It will be convenient to write Eqs. (4,5) as a function of time $t$, by Fourier transforming them:

$$
\begin{aligned}
& \frac{\widehat{g_{R}}}{|g|^{2}}(t)=\widehat{I_{1}}(t) \\
& \frac{\widehat{n}}{|g|^{2}}(t)=\widehat{I_{2}}(t)
\end{aligned}
$$

where $\widehat{I_{1}}$ is the Fourier transform of the real part of $I_{1}$ and $\widehat{I_{2}}$ is the Fourier transform of $I_{2}$

$$
\begin{gathered}
\widehat{I_{1}}(t)=2 \pi P \int_{0}^{\pi} d \theta \sin ^{d-2} \theta \int_{0}^{\infty} d q \cos \theta(\cos \theta-q) q^{2 z-3} \widehat{g_{R}}\left(t r^{z}\right) \widehat{n}\left(t q^{z}\right), \\
\widehat{I_{2}}(t)=\pi P \int_{0}^{\pi} d \theta \sin ^{d-2} \theta \int_{0}^{\infty} d q(\cos \theta-q)^{2} q^{2 z-3} r^{-(d+4-2 z)} \widehat{n}\left(t r^{z}\right) \widehat{n}\left(t q^{z}\right) .
\end{gathered}
$$

Assuming an exponential, or stretched exponential decay for $\widehat{n}(t)$, we look for an asymptotic solution for $t \rightarrow \infty$ of Eq. (7) in the form

$$
\widehat{n_{\infty}}(t)=A(B t)^{\frac{\gamma}{z}} e^{-|B t|^{\frac{\alpha}{z}}}
$$

We will show that such a solution exists and we will determine the exponents $\gamma=(d-1) / 2$ and $\alpha=1$. Even though the scaling part of the correlation and response function are coupled in the two Eqs. (6) and (7), we will see that the specific form of $\widehat{g}(t)$ is not relevant to the large $t$ behaviour of $\widehat{n}(t)$. We will proceed as follows: we evaluate the right hand side of Eq. (7) in the large $t$ limit by saddle point methods. The result of this analysis shows that any $\alpha \leq 1$ allows to reproduce the exponential factor. For $\alpha \leq z$, we can then assume that the left hand side of Eq. (7) is dominated by $g(0)^{-2} \widehat{n}(t)$ (see below), thus the asymptotic form of $I_{2}(t)$ has to be matched with $g(0)^{-2} \widehat{n_{\infty}}(t)$. The exponential factor can be matched by any $\alpha \leq 1$, but we will see that the only value of $\alpha$ that allows also to match the power law factor $t^{\gamma / z}$ is $\alpha=1$. We can then proceed with $\alpha=1$ to get $\gamma=\frac{d-1}{2 z}$ and

$$
A=\frac{g(0)^{-2} 4 \Gamma(4 z-4)}{P 2^{(d-1) / 2} \Gamma((d-1) / 2) \Gamma(2 z-2)^{2}} .
$$


Let us start with the evaluation of the left hand side of Eq. (7). Expanding $|g(x)|^{2}$ in even powers of $x$ around $x=0$, we can write $\left(\left.n \widehat{|| g}\right|^{2}\right)(t)=a_{0} \widehat{n}(t)+a_{1} d^{2} \widehat{n}(t) / d t^{2}+.$. where $a_{0}=g(0)^{-2}$. For $\widehat{n}(t)$ of the form (8) with $\alpha \leq z$, the first term of the series will dominate at large $t$, we can therefore assume that the left hand side of Eq. (7) has the asymptotic behaviour $\left(\left.n \widehat{|| g}\right|^{2}\right)(t) \simeq g(0)^{-2} \widehat{n}(t)$. Let us now turn to the asymptotic analysis of $\widehat{I_{2}}(t)$. First note that the integral $\widehat{I_{2}}(t)$, is symmetric in the exchange $q \rightarrow r, \theta \rightarrow \phi$, where $r \sin \phi=q \sin \theta$ (see Fig 1). This allows us to rewrite $\widehat{I}_{2}(t)$ as twice the integral restricted to the region $q \cos \theta<1 / 2$ :

$$
\widehat{I_{2}}(t)=2 \pi P \int_{0}^{\infty} d q \int_{\bar{\theta}}^{\pi} d \theta \sin ^{d-2} \theta \int_{0}^{\infty} d q(\cos \theta-q)^{2} q^{2 z-3} r^{-(d+4-2 z)} \widehat{n}\left(t r^{z}\right) \widehat{n}\left(t q^{z}\right),
$$

where $\cos \bar{\theta}=\max [1 / 2 q, 1]$. In $\widehat{I_{2}}(t)$ the function $\widehat{n}(t)$ appears in the integrand with the arguments $t q^{z}, t r^{z}$. For large enough $t$, we can always safely replace $\widehat{n}\left(t^{z}\right)$ with its asymptotic form $\widehat{n_{\infty}}\left(t r^{z}\right)$, since in the new region of integration $r \geq 1 / 2$. We have to use more care with $\widehat{n}\left(t q^{z}\right)$, since such replacement is not allowed for all $q$, but only for $q>C / t^{1 / z}$, where $C$ is a large constant. We then have $\widehat{I_{2}}(t) \simeq \widehat{I_{2}^{\infty}}(t)=J_{1}(t)+J_{2}(t)$, where

$$
\begin{aligned}
& J_{1}(t)=2 \pi P \int_{0}^{C / t^{1 / z}} d q \int_{\bar{\theta}}^{\pi} d \theta \sin ^{d-2} \theta(\cos \theta-q)^{2} q^{2 z-3} r^{-(d+4-2 z)} \widehat{n_{\infty}}\left(t r^{z}\right) \widehat{n}\left(t q^{z}\right), \\
& J_{2}(t)=2 \pi P \int_{C / t^{1 / z}}^{\infty} d q \int_{\bar{\theta}}^{\pi} d \theta \sin ^{d-2} \theta(\cos \theta-q)^{2} q^{2 z-3} r^{-(d+4-2 z)} \widehat{n_{\infty}}\left(t r^{z}\right) \widehat{n_{\infty}}\left(t q^{z}\right) .
\end{aligned}
$$

The contribution from $J_{1}$ is negligible with respect to $J_{2}$ as $t \rightarrow \infty$. Let us evaluate an asymptotic expression for $J_{2}$ and postpone the discussion of $J_{1}$ to the end. Inserting $\widehat{n_{\infty}}(t)=A(B t)^{\gamma / z} \exp \left(-|B t|^{\alpha / z}\right)$ in $J_{2}(t)$ gives

$$
J_{2}(t)=2 \pi P A^{2}(B t)^{\frac{2 \gamma}{z}} \int_{C / t^{1 / z}}^{\infty} d q \int_{\bar{\theta}}^{\pi} d \theta \sin ^{d-2} \theta(\cos \theta-q)^{2} q^{2 z-3+\gamma} r^{-(d+4-2 z)+\gamma} e^{-(B t)^{\frac{\alpha}{z}}\left(q^{\alpha}+r^{\alpha}\right)} .
$$

It is immediately clear that the main contribution to this integral will come from the region where $f(q, \theta)=q^{\alpha}+r(q, \theta)^{\alpha}$ has its minimum, i.e. from the segment $\theta=0, C / t^{1 / z}<q<1 / 2$ (for large enough $t, C / t^{1 / z}<1 / 2$ ). The value of $q$ which minimises $f$ will depend on $\alpha$ : for $\alpha<1, f$ reaches its minimum at $q_{0}=C / t^{1 / z}$, where $f\left(q_{0}, 0\right)=C^{\alpha} / t^{\alpha / z}+\mid$ $\left.\left(1-C / t^{1 / z}\right)\right|^{\alpha} \simeq 1+\left(C / t^{1 / z}\right)^{\alpha}+O\left(C / t^{1 / z}\right)$. For $\alpha>1$, the minimum is realized at $q=1 / 2$, where $f(1 / 2,0)=1 / 2^{\alpha-1}$. For $\alpha=1$, all $q$ in the region $C / t^{1 / z}<q<1 / 2$ contribute equally, giving $f(q, 0)=1$. The saddle point approximation in the angular integral gives:

$$
J_{2}(t) \simeq 2 \pi P A^{2}(B t)^{\frac{2 \gamma}{z}} \int_{C / t^{1 / z}}^{1 / 2} d q e^{-(B t)^{\frac{\alpha}{z}}\left(q^{\alpha}+|1-q|^{\alpha}\right)}|1-q|^{2-(d+4-2 z)+\gamma} q^{2 z-3+\gamma} \int_{0}^{\pi} d \theta \theta^{d-2} e^{-(B t)^{\frac{\alpha}{z}} \frac{\alpha}{2} q|1-q|^{\alpha-1} \theta^{2}} .
$$

Performing the integral over $\theta$ in the large $t$ limit:

$$
J_{2}(t) \simeq \pi P A^{2} \Gamma\left(\frac{d-1}{2}\right)\left(\frac{2}{\alpha}\right)^{\frac{d-1}{2}}(B t)^{\frac{2 \gamma}{z}-\frac{d-1}{2 z}} \int_{C / t^{1 / z}}^{1 / 2} d q e^{-(B t)^{\frac{\alpha}{z}}\left(q^{\alpha}+|1-q|^{\alpha}\right)}|1-q|^{\phi} q^{\psi},
$$

where $\phi=2 z-d-2+\gamma+(1-\alpha) \frac{d-1}{2}$ and $\psi=2 z-3+\gamma-\frac{d-1}{2}$. The leading contribution for large $t$ is then given by

$$
J_{2}(t) \simeq \pi P A^{2} \Gamma\left(\frac{d-1}{2}\right)\left(\frac{2}{\alpha}\right)^{\frac{d-1}{2}}(B t)^{\frac{2 \gamma}{z}-\frac{d-1}{2 z}} \begin{cases}\frac{\Gamma\left(\frac{\psi+1}{\alpha}\right)}{\alpha} e^{-(B t)^{\frac{\alpha}{z}}}(B t)^{-\frac{\psi+1}{z}} & \alpha<1 \\ I e^{-(B t)^{\frac{1}{z}}} & \alpha=1 \\ \sqrt{\pi} 2^{-(\phi+\psi)} e^{-\frac{(B t)}{2^{\alpha-1}}(B t)^{-\frac{\alpha}{2 z}}} & \alpha>1\end{cases}
$$

where $I=\int_{0}^{1 / 2} d q q^{\psi}(1-q)^{\phi}$. For $\alpha \leq 1$, the left hand side of Eq. (7) will be dominated by a term of the form $g(0)^{-2} \widehat{n_{\infty}}(t)$, thus $\widehat{I_{2}(t)} \simeq J_{2}(t)$ must be proportional to $\widehat{n_{\infty}}(t)$. This can only be achieved with $\alpha=1$ and $\gamma=\frac{d-1}{2}$. For $\alpha<1$, the stretched exponential factor is recovered, but trying to match the power of $t$ leads to $\gamma=\frac{d-1}{2}+\psi+1$, which can only be satisfied by the unphysical value $z=1$. For $1<\alpha<z$, the the left hand side of Eq. (7) cannot be matched by $J_{2}$. We then conclude that $\alpha$ must be equal to 1 , and the asymptotic solution is then given by Eq. (8). The coefficient $A$ can be determined as well by observing that $\gamma=\frac{d-1}{2}$ gives $\psi=\phi=2 z-3$, and thus $I=\Gamma(2 z-2)^{2} / 2 \Gamma(4 z-4)$ which leads to Eq. (9). 
Let us now go back to the integral $J_{1}$. The argument of $\widehat{n}\left(t q^{z}\right)$ in $J_{1}$ runs between 0 and $C^{z}$. Thus we can obtain an upper bound on $J_{1}$ by replacing $\widehat{n}\left(t q^{z}\right)$ by its maximum in this region (which is given by $\widehat{n}(0)$ if $\widehat{n}(t)$ is a monotonically decreasing function). An analysis similarly to the one done for $J_{2}$, can then be performed for $J_{1}$, leading to $J_{1} \propto(B t)^{\frac{\gamma}{z}-\frac{d-1}{2 z}} e^{-(B t)^{\frac{\alpha}{z}}}$. Thus the contribution to $\widehat{I_{2}^{\infty}}$ from $J_{1}$ is down by a factor $(B t)^{-\frac{\gamma}{z}}$.

The scale parameter $B$ is just that, and remains unfixed in terms of the parameters of the KPZ equation. In a recent work [17], we proposed a scaling ansatz for the correlation function as $z \rightarrow 2$. If such an ansatz is correct, in a parametrization where $g(0)$ is finite the scale $B^{-1}$ on which $t$ varies would go to zero as $z$ approaches 2: $B^{-1} \simeq(2-z)$. Thus the asymptotic solution that we have found here could be read as an asymptotic solution for all $t$ as $z$ approaches 2 .

In summary, we have presented an analytical derivation of an asymptotic solution of the mode-coupling equations for the strong coupling regime of the KPZ equation in the large $t$ limit. We predict a stretched exponential relaxation for the correlation function, with a power law prefactor. We hope that this prediction will stimulate numerical investigations of long-time limits generally.

The authors acknowledge the support of EPSRC under grants GR/L38578 and GR/L97698.

[1] M. Kardar, G. Parisi, and Y-C. Zhang, Phys. Rev. Lett. 56, 889 (1986).

[2] D. Forster, D.R. Nelson, and M.J. Stephen Phys. Rev. A 16, 732 (1977).

[3] D.A. Huse, C.L. Henley, and D.S. Fisher, Phys. Rev. Lett. 55, 2924 (1985); M. Kardar and Y-C. Zhang Phys. Rev. Lett. 58, 2087 (1987).
[4] J.Z. Imbrie and T. Spencer, J. Stat. Phys. 52, 609 (1988); J. Cook and B. Derrida, Europhys. Lett. 10, 195 (1989); J. Cook and B. Derrida, J. Phys. A 23 , 1523 (1990); M.R. Evans and B. Derrida, J. Stat. Phys. 69, 427 (1992).

[5] G. Parisi J. Phys (Paris) 51,1595 (1990); M.Mezard J. Phys (Paris) 51,1831 (1990)

[6] For a review see J. Krug and H. Spohn in Solids far from equilibrium: Growth morphology and defects, ed. by C. Godreche (Cambridge Univ. Press, Cambridge, 1992); A.-L. Barabasi and H. E. Stanley Fractal Concepts in Surface Growth (Cambridge Univ. Press, Cambridge, 1995); T. Halpin-Healy and Y-C. Zhang Phys. Rep. 254, 215 (1995).

[7] W. Götze, in Liquids, Freezing, and the Glass Transition, ed. J.P. Hansen, D. Levesque, and J. Zinn-Justin (Nort Holland, 1991). See also W. Götze and L. Sjögren, Rep. Prog. Phys. 55, 241 (1992).

[8] K. Kawasaki, Ann. Phys. (N. Y.) 61, 1 (1970); K. Kawasaki, in Phase Transitions and Critical Phenomena, Vol 5a, ed. C. Domb and M. S. Green (Academic Press, New York, 1976).

[9] E. Frey and F. Schwabl, Adv. Phys. 43, 577 (1994).

[10] J.P. Doherty, M.A. Moore, J.M. Kim, and A. Bray, Phys. Rev. Lett. 72, 2041 (1994).

[11] R. Kohlrausch, Poggendorf's Ann. Phys. 91, 179 (1854).

[12] G. Williams and D. C. Watts, Trans. Faraday Soc. 66, 80 (1970).

[13] V. Chamberlin, G. Mozurkewich, and R. Orbach, Phys. Rew. Lett. 52, 867 (1984); F. Mezei and A. P. Murani, J. Magn. Mater. 14, 211 (1979).

[14] N. D. Donsker and S.R.S. Varadhan, Commun. Pure Appl. Math. 32, 721 (1979); A. Bunde, S. Havlin, J. Klafter, G. Gräff, and A. Shehter, Phys. Rev. Lett. 78, 3338 (1998); R.M.C. de Almeida, N. Lemke, and I.A. Campbell cond-mat/0003351

[15] L. H. Tang Phys Rev. A 45, 7162 (1992); T. Ala-Nissila, T. Hjelt, J.M. Kosterlitz, and O. Venäläinen, J. Stat. Phys. 72, 207 (1993), E. Marinari, A. Pagnani and G. Parisi cond-mat/0005105.

[16] M. Marsili, A. Maritan, F. Toigo, and J.R. Banavar Rev. Mod. Phys. 68, 963 (1996).

[17] F. Colaiori and M. A. Moore, cond-mat/0010410.

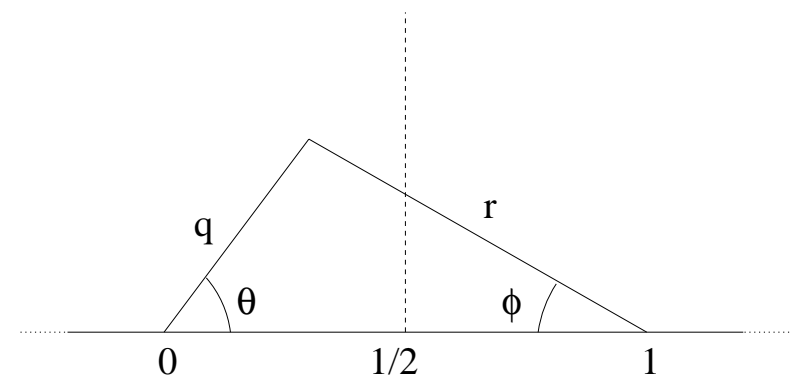

FIG. 1. Region of integration for $\widehat{I_{2}}(t)$ in Eq. 10 ). 